\title{
Die hypothetische Einwilligung im Arzthaftungsprozess
}

\section{Marcel Lanz}

Dr. iur., Rechtsanwalt bei Schärer Rechtsanwälte, Aarau

Verläuft eine ärztliche Behandlung nicht wie gewünscht, kann der Patient geneigt sein, eine mangelhafte Aufklärung zu rügen. Bei einer nachweislich mangelhaften Aufklärung steht dem Arzt die Einrede der hypothetischen Einwilligung offen. Ihr Nachweis ist schwierig, denn das Bundesgericht würdigt sie unter subjektiven Gesichtspunkten des konkret betroffenen Patienten.

\section{Aufklärung und Einwilligung bei der ärztlichen Behandlung}

Jeder Eingriff in die körperliche Integrität ist widerrechtlich, auch die ärztliche Behandlung. Die nach der Aufklärung erfolgte Einwilligung des Patienten macht den ärztlichen Eingriff rechtmässig. Es obliegt dem Arzt zu beweisen, dass er den Patienten ausreichend aufgeklärt und dieser in den Eingriff eingewilligt hat [1]. Massgebend ist das Aufklärungsbedürfnis des betroffenen Patienten [2]. Hat der Arzt den Patienten nicht oder mangelhaft aufgeklärt, kann sich der Arzt auf eine hypothetische Einwilligung des Patienten berufen. Dazu muss er nachweisen, dass der Patient bei gehöriger Aufklärung in die Behandlung eingewilligt hätte. Das Bundesgericht hat die Voraussetzungen in einem Grundsatzentscheid aus dem Jahr 1991 umschrieben [3].

\section{Die Rechtsprechung zur hypothetischen Einwilligung}

Das Bundesgericht hielt fest, dass der Arzt eine hypothetische Einwilligung beweisen müsse. Der Nachweis scheitere, wenn der Patient glaubhaft mache oder wenigstens persönliche Gründe anführe, warum er sich einer Operation widersetzt hätte, insbesondere wenn er ihre Risiken gekannt hätte. Gemäss Bundesgericht darf bei der Beurteilung der Einwände des Patienten nicht auf ein abstraktes Modell eines vernünftigen $\mathrm{Pa}$ tienten abgestellt werden. Massgebend sei die persönliche und konkrete Situation des betroffenen Patienten (sog. subjektive Methode). Es ist somit unbeachtlich, ob ein vernünftiger und besonnener Patient nach erfolg- ter Aufklärung seine Einwilligung in den Eingriff verweigert hätte. Der Schutz des Selbstbestimmungsrechts des Patienten ist ausschlaggebend. Nur wenn der Patient keine persönlichen Gründe geltend macht, aufgrund deren er die Behandlung abgelehnt hätte, beurteilt der Richter, ob die Ablehnung vom Standpunkt eines vernünftigen Patienten aus nachvollziehbar ist [4]. Die soeben umrissene Rechtsprechung hat das Bundesgericht verschiedentlich bestätigt, letztmals im Jahr 2019 [5].

\section{Nachteile der subjektiven Methode für Ärzte}

Die Rechtsprechung des Bundesgerichts, welche in erster Linie den Patientenwillen ins Zentrum rückt, verträgt sich nicht immer mit den Gegebenheiten in der Praxis. Kommt es bei der hypothetischen Einwilligung auf die Situation des konkreten und nicht auf einen vernünftig handelnden Patienten an, kann der Patient versucht sein, die Einrede der hypothetischen Einwilligung ungerechtfertigt zu verhindern, was folgende Überlegung zeigt: Ein Patient, bei dem das Behandlungsergebnis (trotz lege artis ausgeführter Behandlung) nicht wunschgemäss ist, kann zunächst eine mangelnde resp. mangelhafte Aufklärung rügen. Trifft dies zu, haftet der Arzt für sämtliche Schäden - auch bei einer lege artis ausgeführten Behandlung [6]. Die bundesgerichtlichen Anforderungen an die Aufklärung und deren Nachweis sind sehr hoch. Misslingt dem Arzt (deshalb) der Nachweis, dass er den Patienten korrekt und ausreichend aufgeklärt hat, lässt das Bundesgericht dem Arzt wie erwähnt zwar einen Ausweg über die hypothetische Einwilligung offen. Problematisch ist indes, dass der Patient, der mit dem Ergebnis 
der Behandlung nicht zufrieden ist, kaum zugibt, dass er auch in Kenntnis der Risiken (die sich ja möglicherweise im konkreten Fall verwirklicht haben) in die Behandlung eingewilligt hätte [7].

Dass diese Befürchtung nicht von der Hand zu weisen ist, zeigen verschiedene Urteile: So machte ein Patient, der zuvor eine mangelnde Aufklärung gerügt hatte, geltend, bei gehöriger Aufklärung hätte er in die Operation zwar gleichwohl eingewilligt, sie jedoch von einem Spezialisten und nicht vom beklagten Arzt durchführen lassen [8]. Ein Blick in die deutsche Rechtsprechung - an welcher sich das Bundesgericht bei der hypothetischen Einwilligung orientiert - zeigt, dass dieser Einwand des Patienten dem Nachweis einer hypothetischen Einwilligung durch den Arzt meist wirksam entgegensteht [9].

Die Schwierigkeiten einer subjektiven Betrachtungsweise zeigen sich eindrücklich an einem Sachverhalt, den der österreichische Oberste Gerichtshof (OGH) zu entscheiden hatte: Eine Patientin, die beim Tragen einer Metallgerüstprothese im Mund Schmerzen hatte,

\section{Jeder Eingriff in die körperliche Integrität ist} widerrechtlich, auch die ärztliche Behandlung.

die auf einer atypischen Somatisierungsstörung gründeten, machte zunächst geltend, die Behandlung sei nicht lege artis erfolgt. Nachdem der behandelnde Arzt diesen Einwand im erstinstanzlichen Verfahren entkräften konnte, rügte die Patientin eine mangelhafte Aufklärung über das bei ihr erhöhte Risiko chronischer Schmerzen. Die österreichischen Gerichte haben sich soweit ersichtlich mit der Rüge mangelnder Aufklärung nicht eingehend befasst und stattdessen von vornherein auf eine hypothetische Einwilligung geschlossen. Den von der Patientin vor dem OGH dagegen erhobenen Einwand, sie hätte auch nach umfassender Aufklärung über die Risiken nicht in die Behandlung eingewilligt, verwarf der OGH. Der OGH führte insbesondere an, dass sich die Patientin in jedem Fall der Behandlung unterzogen hätte, da eine Nichtbehandlung vernünftigerweise nicht in Betracht gekommen sei [10]. Um zu diesem Ergebnis zu gelangen, wich der OGH von einer subjektiven auf eine objektive Betrachtungsweise aus, indem er sich auf das Verhalten einer vernünftig handelnden Patientin stützte.

Die Problematik einer subjektiven Würdigung der hypothetischen Einwilligung akzentuiert sich bei neuartigen Behandlungsmethoden, welche der Arzt an Stelle einer etablierten anwendet. In diesen Fällen erscheint das Abstützen auf die individuellen Empfindungen (die ja bloss hypothetischer Natur sind) unsachgemäss. Hat der Arzt einen ängstlichen, eher risikoaver- sen Patienten behandelt, wäre ihm die Berufung auf eine hypothetische Einwilligung faktisch verwehrt, da dieser Patient wohl kaum in eine neuartige Behandlungsmethode einwilligen würde. Die deutsche Rechtsprechung, die dem Schweizer Bundesgericht oftmals als Leitlinie dient, zeigt sich bei der hypothetischen Einwilligung im Falle neuer Behandlungsmethoden indes sehr patientenfreundlich. Der deutsche Bundesgerichtshof senkte gar die Anforderungen an den Nachweis des Patienten, dass er bei gehöriger Aufklärung nicht in eine neuartige Behandlung eingewilligt hätte [11].

\section{Teilweise Berücksichtigung objektiver Kriterien}

Die Problematik einer subjektiven Würdigung bei der hypothetischen Einwilligung haben US-amerikanische Gerichte seit längerem erkannt und entsprechende Korrekturen vorgenommen. Die Gerichte haben sich von einer rein subjektiven Würdigung der Umstände abgewandt und berücksichtigen das Verhalten eines hypothetisch vernünftig handelnden Patienten [12].

Dass sich das Bundesgericht einer objektiven Würdigung - sozusagen als nachgelagertes Korrektiv zur subjektiven Beurteilung - nicht ganz verschliesst, zeigen zwei neuere Urteile. Obwohl das Bundesgericht im ersten Entscheid in Anwendung der subjektiven Methode zum Schluss gelangt ist, dass eine hypothetische Einwilligung zu verneinen ist, prüfte es die Einwände des Patienten anschliessend, ob diese als allgemein vernünftig erscheinen [13]. In einem früheren

Auf jeden Fall ist aber davon abzuraten, vorschnell den Einwand einer hypothetischen Einwilligung des Patienten zu erheben.

Entscheid war das Bundesgericht zum Schluss gelangt, dass eine Ablehnung der Behandlung vernünftigerweise nicht in Betracht kam, weshalb eine hypothetische Einwilligung vorliege [14]. Auch die deutsche Rechtsprechung lässt es vermehrt zu, die Einwände des Patienten auf ihre Plausibilität - durch Vergleich mit einem vernünftig handelnden Patienten - zu prüfen [15]. Die Berücksichtigung objektiver Kriterien macht die gerichtliche Beurteilung für den Arzt vorhersehbarer und stärkt die Rechtssicherheit.

\section{Die Geltendmachung der hypothetischen Einwilligung im Prozess}

Abschliessend bleibt festzuhalten, dass die ärztliche Aufklärung generell nach höchster Sorgfalt verlangt. 
Nur so vermeidet der Arzt, für die Verwirklichung allfälliger Risiken belangt zu werden. Denn selbst bei einer lege artis durchgeführten Behandlung, die aber aufgrund einer mangelhaften Aufklärung erfolgt, ist der Arzt für allfällige Schäden haftbar. Die Entlastung mit Hilfe der hypothetischen Einwilligung ist als letzter Ausweg mit vielen Unwägbarkeiten verbunden.

Bringt der Arzt im Haftpflichtprozess den Einwand der hypothetischen Einwilligung vor, erscheint es sinnvoll, sowohl subjektive als auch objektive Gründe $\mathrm{zu}$ nennen, die nahelegen, dass der Patient auch bei vollständiger Aufklärung in die Behandlung eingewilligt hätte. Dabei ist einzelfallweise zu beurteilen, in welchem prozessualen Stadium die entsprechenden Einwände erhoben werden: Macht der Arzt bereits in der Klageantwort sowohl subjektive als auch objektive Gründe für das Vorliegen einer hypothetischen Einwilligung geltend, muss der Patient zu beiden Vorbringen Stellung beziehen.

Auf jeden Fall ist aber davon abzuraten, vorschnell den Einwand einer hypothetischen Einwilligung des $\mathrm{Pa}$ tienten zu erheben. Denn damit entsteht der Eindruck, die Aufklärung sei tatsächlich mangelhaft erfolgt.

\section{Die Rechtssicherheit stärken}

Die vom Bundesgericht postulierte subjektive Methode erscheint zwar patientenfreundlich. Objektive Kriterien sollten indes auch in der Schweiz stärker berücksichtigt werden, um für die Ärzte eine einheitlichere und vor allem vorhersehbare Praxis zu schaffen. Dies würde schlussendlich die Rechtssicherheit stärken.

\section{Literatur}

1 Statt vieler: BGE 133 III 121, E. 4.1.1.

2 BGE 117 Ib 197, E. 3b.

3 BGE 117 Ib 197, E. 5.

4 BGE $117 \mathrm{Ib}$ 197, E. $5 \mathrm{C}$

5 BGE 133 III 121, E. 4; BGer 4A_353/2018 vom 1.4.2019, E. 2.

6 BGE 133 III 121, E. 4.1.1.

7 In BGer 4A 353/2018 vom 1.4.2019 (Sachverhalt).

8 BGer 4A_453/2014 vom 23.2.2015, E. 6.3.

9 Deutsch E, Spickhoff A. Medizinrecht, 7. Aufl., Heidelberg 2014, N 522 .
10 OGH 1 Ob39/16s vom 31.3.2016.

11 Münchener Kommentar BGB-Wagner, §630h N 51 mit weiteren Hinweisen.

12 Lanz M. Die Haftung beim medizinischen Einsatz synthetischer Nanopartikel, Diss. Fribourg 2020, N 631

13 BGer 4A 453/2014 vom 23.2.2015, E. 6.3.

14 BGer 4A_98/2010 vom 1.4.2010

15 MK BGB-Wagner, §630h N 50; Deutsch/Spickhoff, N 523.

\section{Das Wichtigste in Kürze}

- Hat der Patient den Eindruck, er sei vor einer ärztlichen Behandlung unzureichend aufgeklärt worden oder ist das Ergebnis der Handlung nicht wie gewünscht, kann er eine mangelhafte Aufklärung rügen.

- Der Arzt kann sich hierbei auf die hypothetische Einwilligung des Patienten berufen. Diese muss vom Arzt bewiesen werden.

- Gemäss Bundesgericht darf bei der Beurteilung der Einwände des Patienten nicht auf einen vernünftig handelnden Patienten abgestellt werden. Massgebend ist die persönliche und konkrete Situation des betroffenen Patienten (sog. subjektive Methode).

- Der Autor spricht sich dafür aus, dass objektive Kriterien, das heisst die Annahme eines hypothetisch vernünftig handelnden Patienten, stärker berücksichtigt werden sollten. So soll eine einheitlichere und vorhersehbare Praxis geschaffen und die Rechtssicherheit gestärkt werden.

\section{L'essentiel en bref}

- Si le patient a l'impression de ne pas avoir été suffisamment informé avant le traitement, ou si le résultat du traitement n'est pas celui attendu, il peut se plaindre d'une information insuffisante.

- Le médecin peut s'appuyer sur le consentement hypothétique du patient, dont il doit apporter les preuves

- Selon le Tribunal fédéral, les contestations du patient ne peuvent pas être évaluées sur la base du caractère raisonnable de ce dernier. La situation personnelle et concrète du patient est déterminante (méthode dite subjective).

- L'auteur estime qu'il faudrait tenir davantage compte des critères objectifs, c'est-à-dire du postulat d'un patient agissant de manière supposément raisonnable. Cela devrait engendrer une pratique plus uniforme et prévisible et renforcer la certitude juridique. 